3. - Chain transforms, Proc. Amer. Math. Soc. vol. 5 (1954) pp. 677-688.

4. H. Kober, On fractional integrals and derivatives, Quart. J. Math. Oxford Ser. (2) vol. 11 (1940) pp. 193-211.

5. E. C. Titchmarsh, Introduction to the theory of Fourier integrals, Oxford, 1937.

6. E. T. Whittaker and G. N. Watson, Modern analysis, Cambridge, 1915.

7. D. Widder, The Laplace transform, Princeton, 1946.

McGill University

\title{
SUR LES FONCTIONS DERIVEES, INTÉGRABLES AU SENS DE RIEMANN ET SUR LES DERIVEES PARTIELLES MIXTES
}

\section{S. MARCUS}

1. Introduction. D'après A. P. Morse et W. W. Bledsoe, une fonction $f: S \rightarrow T$ (où $S$ et $T$ sont deux espaces métriques avec les distances $d$ et $d^{\prime}$ ) est dite envoisinée (neighborly) au point $x \in S$, si pour chaque $\epsilon>0$ il existe une sphère non vide et ouverte $U \subset S$, telle que pour tout $y \in U$ on ait $d(x, y)+d^{\prime}(f(x), f(y))<\epsilon,[1]$. Puisque $U$ n'est pas soumise à la condition de contenir le point $x$, la propriété ci-dessu est une généralisation de la notion de continuité. A. V. Martin énonce dans [7] (sans démonstration) le théorème suivant: "Si $f$ est une fonction réelle d'une variable réelle et si la dérivée $f^{\prime}$ existe et est intégrable au sens de Riemann sur tout intervalle compact, alors $f^{\prime}$ est envoisinée en chaque point." A. V. Martin affirme en [7] que ce théorème pourrait donner certaines suggestions concernant le problème, encore non résolu, de trouver une caractérisation intrinsèque des fonctions dérivées.

Dans la note présente, en utilisant l'équivalence de la notion de "fonction envoisinée" avec une notion introduite en 1933 par S. Kempisty (Théorème 1), on établit un théorème qui contient comme un cas particulier le résultat de A. V. Martin (Théorème 2). Puis, par certains exemples, on montre que la propriété d'être envoisinée est loin d'appartenir à chaque fonction dérivée, même bornée (Théorème 4), mais elle peut appartenir à une dérivée bornée qui n'est pas intégrable au sens de Riemann (Théorème 3 ). Etant donnée la liaison étroite entre les fonctions dérivées et les fonctions approximativement continues, on établit, pour ces dernières, un cas où elles sont envoisinées (Théorème 5). A la fin on donnent quelques applications au problème de la commutativité des dérivées partielles mixtes (Théorèmes 6 et 7).

Received by the editors March 26, 1958. 
2. On dit, d'après S. Kempisty, que $f: S \rightarrow T$ est quasicontinue en $x \in S$, si pour chaque sphère $U \subset S$, centrée en $x$, et pour chaque sphère $V \subset T$, centrée en $f(x)$, il existe une sphère $W \subset U$ telle que $f(W) \subset V[\mathbf{4}]$.

THÉORÈME 1. $f$ est envoisinée en $x \in S$ si et seulement si elle est quasicontinue en $x$.

Démonstration. Si $f$ est envoisinée en $x$, alors il existe, pour $\epsilon>0$, une sphère $D_{n} \subset S$, telle que $d(x, y)+d^{\prime}(f(x), f(y))<\epsilon / n$, pour chaque $y \in D_{n}$. Considérons une sphère ouverte $D$ contenant $x$. Puisque $d(x, y)<\epsilon / n$ pour chaque $y \in D_{n}$, il s'ensuit que, pour $n$ assez grand, on a $D_{n} \subset D$. Mais on a aussi, pour chaque $y \in D_{n}, d^{\prime}(f(x), f(y))<\epsilon$, donc $f$ est quasicontinue en $x$.

Si $f$ est quasicontinue en $x$, alors, pour $\epsilon>0$ et désignant par $D$ la sphère centrée en $x$ et de rayon $\epsilon / 2$, il existe une sphère $D_{1} \subset D$, telle que $d^{\prime}(f(x), f(y))<\epsilon / 2$ pour chaque $y \in D_{1}$. Mais on a aussi, pour $y \in D_{1}, d(x, y)<\epsilon / 2$, donc $d(x, y)+d^{\prime}(f(x), f(y))<\epsilon$ et $f$ est envoisinée en $x$.

THÉORÈme 2. Une fonction dérivée, continue presque partout sur $(a, b)$, est quasicontinue sur $(a, b)$.

Démonstration. Soient $a<x_{0}<b$ et $\epsilon>0$. Posons $A_{\epsilon}=\left\{x ; f\left(x_{0}\right)\right.$ $\left.-\epsilon / 2<f(x)<f\left(x_{0}\right)+\epsilon / 2\right\}$. Si $f$ est une fonction dérivée sur $(a, b)$, alors, d'après un théorème de Denjoy de [2], un ensemble de la forme $\{x ; k<f(x)<l, a<x<b\}$ est vide ou de mesure positive. Puisque $A_{\epsilon}$ contient, en tout cas, le point $x_{0}$, il s'ensuit que $A_{\epsilon}$ est de mesure positive sur chaque intervalle contenant $x_{0}$. Soit $I$ un tel intervalle. En tenant compte que $f$ est continue presque partout sur $(a, b)$, donc sur $I$, il existe un $\xi \in A_{\epsilon} \cap I$, tel que $f$ soit continue en $\xi$. Il existe donc un intervalle $J \subset I$, centré en $\xi$ et tel que l'oscillation de $f$ sur $J$ soit inférieure à $\epsilon / 2$. On a donc, pour $x \in J,\left|f(x)-f\left(x_{0}\right)\right|$ $\leqq|f(x)-f(\xi)|+\left|f(\xi)-f\left(x_{0}\right)\right|<\epsilon / 2+\epsilon / 2=\epsilon$ et $f$ est quasicontinue en $x_{0}$. Mais $x_{0}$ étant quelconque dans $(a, b)$, il résulte que $f$ est quasicontinue sur $(a, b)$.

Des Théorèmes 1 et 2 on déduit le suivant

Corollaire (Théorème de A. V. Martin). Une fonction dérivée, intégrable au sens de Riemann sur $[a, b]$, est envoisinée sur $[a, b]$.

3. Il est naturel de se poser la question si la condition d'être envoisinée est suffisante pour qu'une fonction dérivée bornée soit intégrable au sens de Riemann. La réponse est donnée par le 
THÉORÈME 3. Il existe une fonction dérivée bornée, envoisinée sur $[0,1]$, qui n'est pas intégrable au sens de Riemann sur $[0,1]$.

Démonstration. Nous allons utiliser un exemple de V. Volterra [9]. Soit $E$ un ensemble parfait, non dense et de mesure positive, construit sur $[0,1]$. Posons $\phi(u, v)=(u-v)^{2} \sin 1 /(u-v)$. Définissons une fonction $F(x)$ comme il suit: Si $x \in E$, alors $F(x)=0$; si $(a, b)$ est un intervalle contigu à $E$ est si $c$ est la plus grande valeur qui annule la dérivée $\phi_{x}^{\prime}(x, a)$ et telle que $a<c \leqq(a+b) / 2$, alors

$$
F(x)=\left\{\begin{array}{cll}
\phi(x, a), & \text { si } a \leqq x \leqq a+c, \\
\phi(a+c, a), & \text { si } a+c<x<b-c, \\
-\phi(x, b), & \text { si } \quad b-c \leqq x \leqq b .
\end{array}\right.
$$

Il est visible que $F^{\prime}(x)$ existe et est continue, donc envoisinée, dans chaque intervalle contigu à $E$ et que la dérivée à droite (resp. à gauche) de $F(x)$ existe et est nulle en chaque extrémité gauche (resp. droite) d'un tel intervalle. Un calcul simple montre que $F(x)$ est dérivable pour chaque $x \in E$ et qu'on a, pour un tel $x, F^{\prime}(x)=0$. Du fait que, en chaque intervalle $(a, b)$, contigu à $E, F^{\prime}(x)$ s'annule une infinité de fois et que les zéros de $F^{\prime}(x)$ s'accumulent en a et en $b$ et en tenant compte de la continuité de $F^{\prime}(x)$ sur $(a, b)$, on déduit que $F^{\prime}(x)$ est quasicontinue en chaque $x \in[0,1]$. D'après le Théorème 1 , il s'ensuit que $F^{\prime}(x)$ est envoisinée sur $[0,1]$. D'autre part, il est aisé de voir que $F^{\prime}(x)$ est bornée sur $[0,1]$ et discontinue en chaque $x \in E$. Mais $E$ est de mesure positive. Il résulte que $F^{\prime}(x)$ n'est pas intégrable au sens de Riemann sur $[0,1]$.

On pourrait soupçonner, en tenant compte du Théorème 3 , que toute fonction dérivée est envoisinée. Ce n'est pas le cas, comme le montre le

THÉoRk̀me 4. Il existe une fonction $f$ dérivée, bornée sur $(a, b)$, telle que l'ensemble des points où $f$ n'est pas envoisinée es de mesure positive dans chaque sous-intervalle de $(a, b)$.

Démonstration. Considérons une fonction $f$ dérivée, bornée, non négative sur $(a, b)$ et telle que chacun des ensembles $\{x ; f(x)=0\}$, $\{x ; f(x)>0\}$ soit partout dense sur $(a, b)$. Un exemple d'une telle fonction est donné dans [8]. D'après le théorème de Denjoy de [2], l'ensemble $\{x ; f(x)>0\}$ est de mesure positive dans chaque sousintervalle de $(a, b)$. En même temps, en chaque point de cet ensemble, $f$ n'est pas quasicontinue, puisque $\{x ; f(x)=0\}$ est partout dense sur $(a, b)$. En tenant compte du Théorème 1, on déduit le Théorème 4 . 
4. On sait qu'il existe une liaison étroite entre les fonctions dérivées et les fonctions approximativement continues. Dans cette ordre d'idées, peut-être il n'est pas dépourvu d'intérêt le

THÉORغ̀me 5. Si f est approximativement continue en chaque point $d e(a, b)$ et continue presque partout sur $(a, b)$, alors $f$ est envoisinée sur $(a, b)$.

Démonstration. Soient $a<x_{0}<b$ et $\epsilon>0$. Du fait que $f$ est approximativement continue sur $(a, b)$ il s'ensuit que l'ensemble $A_{\epsilon}$ $=\left\{x ; f\left(x_{0}\right)-\epsilon / 2<f(x)<f\left(x_{0}\right)+\epsilon / 2\right\}$ est formé seulement de ses points de densité. Ceci entraîne que pour chaque $\epsilon>0$ et pour chaque intervalle $I$ contenant le point $x_{0}$, l'ensemble $A_{\epsilon} \cap I$ est de mesure positive. En suivant une voie analogue à celle utilisée dans la démonstration du Théorème 2 et en tenant compte du Théorème 1 , on déduit que $f$ est envoisinée en $x_{0}$. Mais $x_{0}$ est arbitraire dans $(a, b)$, donc $f$ est envoisinée sur $(a, b)$.

Remarque 1. Pour les fonctions bornées, le Théorème 5 est contenu dans le Théorème 2. En effet, d'après un théorème de Denjoy de [3], une fonction bornée et approximativement continue sur $(a, b)$ est une dérivée sur $(a, b)$ et, d'autre part, en vertu du Théorème 1 , toute fonction quasicontinue est envoisinée.

Remarque 2. Le théorème de $\mathrm{A}$. V. Martin n'est pas contenu dans le Théorème 5 . En effet, il existe une fonction dérivée intégrable au sens de Riemann sur $[a, b]$, qui n'est pas approximativement continue sur $[a, b]$. Considérons pour cela les fonctions

$$
f(x)=\left\{\begin{array}{ll}
x^{2} \sin 1 / x, & \text { si } x \neq 0, \\
0, & \text { si } x=0,
\end{array} \quad \phi(x)= \begin{cases}2 x \sin 1 / x, & \text { si } x \neq 0, \\
0, & \text { si } x=0\end{cases}\right.
$$

et posons $\psi(x)=\phi(x)-f^{\prime}(x)$. On a

$$
\psi(x)= \begin{cases}\cos 1 / x, & \text { si } x \neq 0 \\ 0, & \text { si } x=0\end{cases}
$$

$\psi(x)$, comme différence de fonctions dérivées, est aussi une dérivée. En outre, $\psi(x)$ est bornée et continue pour chaque $x \neq 0$. Donc $\psi(x)$ est intégrable au sens de Riemann sur $[-1,+1]$. D'autre part, il est visible que $\psi(x)$ n'est pas approximativement continue pour $x=0$.

5. Applications au problème de la commutativité des dérivées partielles mixtes. Considérons une fonction réelle, finie, $f$, définie dans un domaine $D \subset R^{n}$ et douée de dérivées partielles mixtes jusqu'à un certain ordre $p$. Convenons de dire qu'une dérivée partielle mixte (d'ordre $\leqq p$ ) est commutative en $x \in D$, si sa valeur en $x$ est indépen- 
dante de l'ordre des dérivations. Si une dérivée partielle mixte est commutative en chaque point de $D$, alors elle est commutative dans $D$. Dans [5] nous avons établi le théorème suivant: "Si les dérivées partielles mixtes, d'ordre $p$, de la fonction $f$, existent et sont continues, en chaque point de $D$, par rapport à chaque variable prise à part, alors chaque dérivée partielle mixte, d'ordre $\leqq p$, est commutative dans $D$." Nous nous proposons, dans ce qui suit, d'améliorer ce résultat. Mais d'abord un

Lemme. Si deux fonctions réelles, finies, $f$ et $g$, définies dans le domaine $D \subset R^{n}$, sont, dans $D$, par rapport à chaque variable prise à part, des fonctions dérivées, presque partout continues, et s'il existe un ensemble $E$, partout dense dans $D$, tel que $f(x)=g(x)$ pour chaque $x \in E$, alors $f(x)=g(x)$ pour chaque $x \in D$.

Démonstration. Supposons qu'il existe $x \in D$, tel que $\phi(x)=f(x)$ $-g(x) \neq 0$. En vertu du Théorème 2, la fonction $\phi$ est, dans $D$, quasicontinue par rapport à chaque variable prise à part. D'après une théorème de $\mathrm{S}$. Kempisty de [4], une telle fonction est quasicontinue dans $D$, donc quasicontinue en $x$. Soit $0<\epsilon<|\phi(x)|$. Il existe une sphère $S \subset D$, telle que $z \in S$ entraîne $|\phi(x)-\phi(z)|<\epsilon$. Mais, $E$ étant partout dense dans $D$, il existe un point $y \in S \cap E$, donc $\phi(y)=0$. On en déduit que $|\phi(x)|=|\phi(x)-\phi(y)|<\epsilon$, ce qui est contradictoire.

THÉORÈme 6. Soit $f$ une fonction réelle, finie, définie dans un domaine $D \subset R^{n}$. Soit $p$ un entier supérieur à 1. Si les dérivées partielles mixtes, d'ordre $p$, de la fonction $f$, existent et sont, dans $D$, par rapport à chaque variable prise à part, des fonctions dérivées, presque partout continues, alors chaque dérivée partielle mixte, d'ordre $\leqq p$, est commutative dans $D$.

Démonstration. La commutativité, dans les conditions du théorème, des dérivées partielles mixtes d'ordre $<p$, n'est pas un fait nouveau. Elle est démontrée en [5]. D'après le Théorème 2, chaque dérivée partielle mixte d'ordre $p$ est, dans $D$, quasicontinue par rapport à chaque variable prise à part. Alors, en vertu d'un théorème de S. Kempisty de [4], chaque dérivée partielle mixte d'ordre $p$ est quasicontinue dans $D$. En vertu du Lemme 1 de [5], une fonction quasicontinue dans $D$ est ponctuellement discontinue dans $D$. Il existe donc un ensemble $B C D$, de première catégorie, tel que chaque dérivée partielle mixte d'ordre $p$ est continue dans $D-B$. D'après le théorème classique de Schwarz, une telle dérivée partielle est commutative dans $D-B$. En tenant compte du lemme ci-dessus, il résulte que cette commutativité a lieu en chaque point de $D$. 
En vertu des Théorèmes 1 et 5 , on constate que le lemme ci-dessus reste valable si le mot "dérivées" est remplacé par "approximativement continues." On parvient ainsi, par un raisonnement similaire au celui utilisé dans la démonstration du Théorème 6 , au

THÉORÈme 7. Soit $f$ une fonction réelle, finie, définie dans un domaine $D \subset R^{n}$. Soit $p$ un entier supérieur à 1 . Si les dérivées partielles mixtes, d'ordre $p$, de la fonction $f$, existent et sont, dans $D$, par rapport à chaque variable prise à part, des fonctions approximativement continues, presque partout continues, alors chaque dérivée partielle mixte, d'ordre $\leqq p$, est commutative dans $D$.

D'autres applications des fonctions quasicontinues au problème de la commutativité des dérivées partielles mixtes se trouvent dans [6].

\section{Ouvrages CItés}

1. W. W. Bledsoe, Neighborly functions, Proc. Amer. Math. Soc. vol. 3 (1952) pp. 114-115.

2. A. Denjoy, Sur une propriêté des fonctions dérivées, Enseignement Math. vol. 18 (1916) pp. 320-328.

3. - Sur les fonctions dérivées sommables, Bull. Soc. Math. France vol. 43 (1915) p. 172.

4. S. Kempisty, Sur les fonctions quasicontinues, Fund. Math. vol. 19 (1932) pp. 184-197.

5. S. Marcus, Sur les dérivées partielles mixtes, C. R. Acad. Sci. Paris vol. 246 (1958) pp. 522-524.

6. - Sur les fonctions quasicontinues au sens de S. Kempisty (à paraltre dans les Fundamenta Math.).

7. A. V. Martin, On derivatives and neighborly functions, Bull. Amer. Math. Soc. vol. 63 (1957) pp. 24-25.

8. D. Pompeiu, Sur les fonctions dérivées, Math. Ann. vol. 63 (1907) p. 326.

9. V. Volterra, Sui principii del Calcolo integrale, Giornale di Matematiche, Napoli vol. 19 (1881) pp. 334-336.

Institutul de Matematic al Academiei R.P.R., Bucharest 